\title{
Porcine Brachial Artery Tortuosity for In Vivo Evaluation of Neuroendovascular Devices
}

\author{
S. Carniato, M. Mehra, R.M. King, A.K. Wakhloo, and M.J. Gounis
}

- $D$

\begin{abstract}
SUMMARY: We report a novel model of arterial tortuosity in the porcine brachial artery for testing of endovascular devices in the flexed forelimb position. This provides an ideal vascular territory for an in vivo assessment of guidewires, microcatheters, and endovascular implants because it closely mimics the challenging curvature at the carotid siphon.
\end{abstract}

ABBREVIATIONS $\mathrm{AC}=$ average curvature; $3 \mathrm{DRA}=3 \mathrm{D}$ rotational angiography; $\mathrm{CCA}=$ common carotid artery

A dvances in interventional neuroradiology are reshaping the treatment paradigms and have facilitated the development of a wide array of endoluminal techniques for vascular lesions that were earlier only approachable neurosurgically. The ICA siphon is extremely relevant for intracranial access, because its tortuosity and bony encasement can be a significant impediment for guidewires and microcatheters to gain intracranial access and may also preclude the delivery of devices like intracranial stents.

As newer devices are developed, they are first evaluated in vitro and in cadaveric models. Although indispensible, these can only assess the mechanical and structural responses. For a realistic simulation of the critical vessel viscoelastic and physiologic responses, an in vivo assessment of endovascular devices is crucial. We describe a porcine brachial artery tortuosity model to provide a challenging vascular territory for endovascular device testing.

\section{TECHNIQUE}

Three Yorkshire swine (male; mean weight, $47 \mathrm{~kg}$ ) were used for the purposes of our study. All procedures were conducted in accordance with the protocol approved by the Institutional Animal Care and Use Committee of University of Massachusetts Medical School. The animals were premedicated with glycopyrrolate $(0.01$

Received May 2, 2011; accepted after revision June 10.

From Research and Development (S.C.), Stryker Neurovascular, Fremont, California; and Department of Radiology (M.M., R.M.K., A.K.W., M.J.G.), New England Center for Stroke Research, University of Massachusetts Medical School, Worcester, Massachusetts.

This work was supported by Stryker Neurovascular.

Please address correspondence to Matthew J. Gounis, PhD, Department of Radiology, University of Massachusetts, New England Center for Stroke Research, 55 Lake Ave N, SA-107R, Worcester, MA 01655; e-mail: matt.gounis@umassmed.edu

- Indicates open access to non-subscribers at www.ajnr.org

$\square$ Indicates article with supplemental on-line video.

http://dx.doi.org/10.3174/ajnr.A2778 $\mathrm{mg} / \mathrm{kg}$ ). Anesthesia was induced by an intramuscular injection of tiletamine (Telazol, $5 \mathrm{mg} / \mathrm{kg}$ ), ketamine $(2.5 \mathrm{mg} / \mathrm{kg})$, and xylazine $(2.5 \mathrm{mg} / \mathrm{kg})$ and maintained with mechanical ventilation of oxygen containing $1 \%-3 \%$ isoflurane. Continuous monitoring of the heart rate, respiration, oxygen saturation level (pulse oximetry), end-tidal carbon dioxide, and temperature allowed real-time assessment of the physiologic status of the animal. A $6 \mathrm{~F}$ hemostatic introducer was placed in the right femoral artery by using a modified Seldinger technique following a cut-down. The introducer was secured in place, with the distal aspect of the femoral artery ligated. A $6 \mathrm{~F}$ catheter was then advanced over the guidewire under $\mathrm{x}$-ray fluoroscopy.

\section{Animal Positioning}

Selective intra-arterial digital subtraction angiography of the right and left subclavian arteries was performed in both the extended and the flexed forelimb position of the swine by using table restrainers, as illustrated in Fig $1 A,-B$. The guide catheter was selectively positioned in the brachial branch of the subclavian artery. A selective intra-arterial injection of the contrast agent (Iopamidol, Isovue; Bristol Myers Squibb, Princeton, New Jersey) was administered with a power injector at an injection rate of $2.5 \mathrm{~mL} / \mathrm{s}$ for 11.2 seconds with a 7-second delay to acquire a 3DRA dataset (Fig $1 C,-D)$.

\section{Image Processing}

AC (the parameter used to describe the vessel tortuosity), path length, and the vessel diameters were determined by using a method previously described. ${ }^{1}$ Briefly, the 3DRA dataset was imported to Mimics (Materialize, Leuven, Belgium). The target tissue was extracted from the neighboring tissue through thresholding. To generate a segmentation mask, we set maximum and minimum threshold values, and the segmentation was further restricted by cropping the mask. The selected pixels in each sec- 


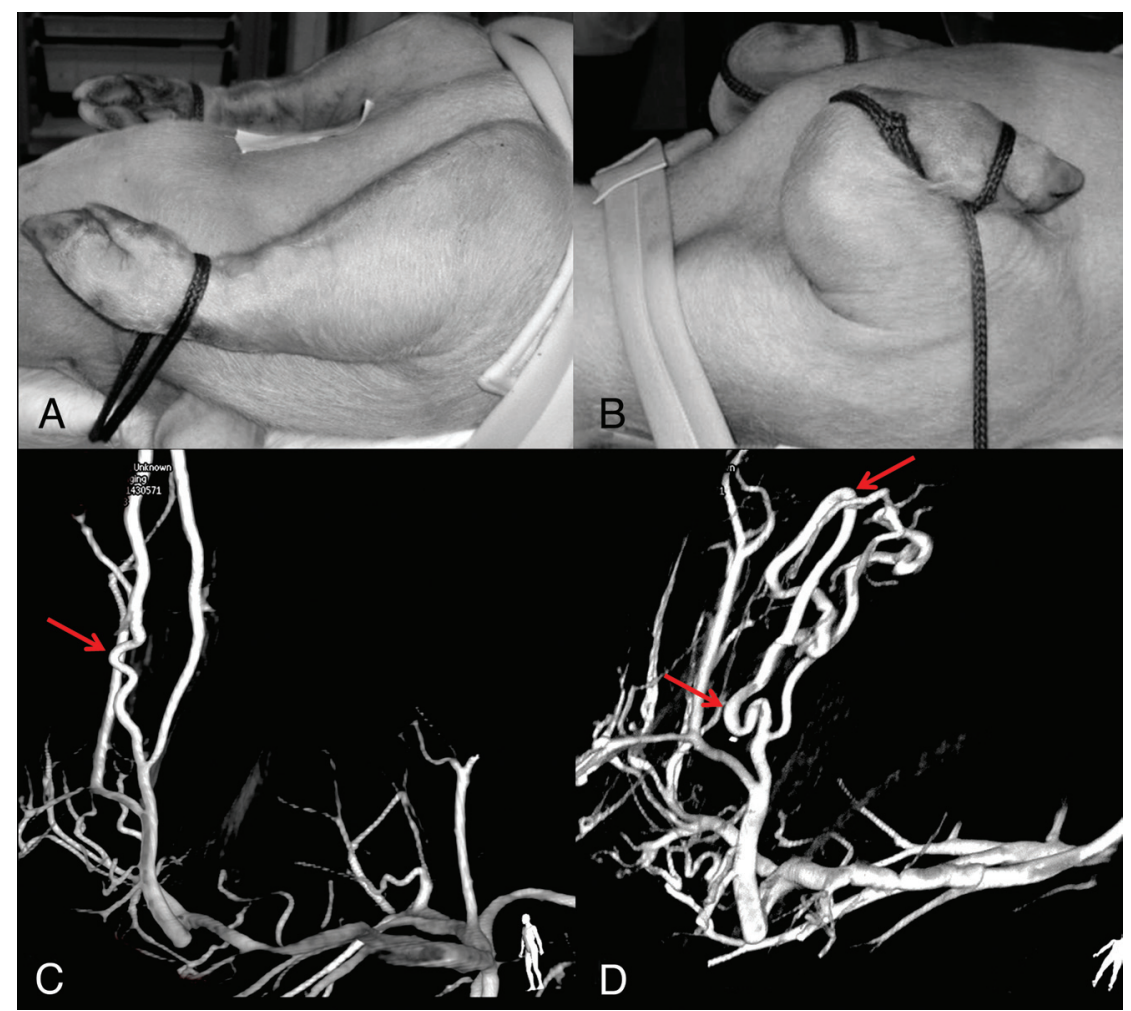

FIG 1. Images depicting the positioning of the swine forelimb and the 3DRA acquired through the guide catheter in the subclavian artery in the extended $(A$ and $C)$ and the flexed ( $B$ and $D)$ position, revealing their arterial tortuosity (arrows). The number of bends and the tortuosity of the path are accentuated in the flexed position.

tion were connected; this step resulted in a clean mask for the use of $3 \mathrm{D}$ model construction. The resulting centerline was composed of control points equally spaced on a constant $0.4-\mathrm{mm}$ interval. The path length of the extracted centerline and the diameter of the best-fit circle to the vessel cross-section at each control point were recorded and averaged.

\section{Statistical Analysis}

The AC, path length, and the vessel diameter of the porcine brachial artery vasculature in the flexed and extended position were compared with a realistic human ICA dataset previously described. ${ }^{1}$ Data normality were tested by using the method described by Kolmogorov and Smirnov. Nonparametric data analysis of the AC and the path length was performed by using the Kruskal-Wallis test, and a parametric analysis for vascular diameters was performed by using a 2-tailed unpaired $t$ test by using GraphPad Prism 5.04 software (GraphPad, San Diego, California).

\section{RESULTS}

The AC, path length, and vascular diameter calculated for the arterial segments (average \pm standard error of the mean) in the extended $\left(0.28 \pm 0.06 \mathrm{~mm}^{-1}, 13.99 \pm 1.66 \mathrm{~mm}\right.$, and $2.60 \pm 0.19$ $\mathrm{mm})$ and the flexed $\left(0.29 \pm 0.02 \mathrm{~mm}^{-1}, 17.59 \pm 0.77 \mathrm{~mm}\right.$, and $2.59 \pm 0.12 \mathrm{~mm}$ ) positions in swine are similar (Fig 2). In comparison, the values calculated for the population-representative human carotid siphon are $0.34 \pm 0.02 \mathrm{~mm}^{-1}, 22.60 \pm 0.79 \mathrm{~mm}$, and $4.15 \pm 0.09 \mathrm{~mm}$, respectively. ${ }^{1}$
The nonparametric Kruskal-Wallis test was performed to compare the means of the AC of the human carotid siphon and the tortuous bends of the porcine brachial artery both in the flexed and the extended position. There was no statistical difference between the groups $(P=$ $.44)$. Also, there was no statistical difference between the path lengths among the 3 groups $(P=.45)$. The vascular diameters between the human and the porcine arteries were, however, significantly different $(P<.0001)$.

The positioning of the forelimb in the flexed position increased the number of tortuous bends in comparison with the extended position (Fig 1, On-line Video) and emphasized the complexity of the curvatures. This arterial territory allows a successful navigation and testing of an array of endovascular devices like guidewires, microcatheters, endovascular stents, and delivery systems.

\section{DISCUSSION}

Forceful catheterization of the carotid siphon can result in thrombosis and vasospasm as a result of endothelial injury. The growing availability of endovascular devices for delivery to the intracranial space creates the need for a more realistic approximation of the tortuosity of the human intracranial vessels. The model described provides a robust environment to challenge the delivery and deployment of neuroendovascular devices.

Although some physical properties of microcatheters and guidewires like radio-opacity and handling can be evaluated in in vitro models, other parameters like steerability/torquability, lubricity, stiffness, and durability are better analyzed in an in vivo environment. Also, the ability to evaluate the consequences of endovascular manipulation like vessel straightening and displacement, vessel vasospasm, endothelial injury, secondary platelet adhesion, fibrin polymerization, and vessel perforation makes in vivo assessment indispensible.

Models simulating the carotid tortuosity have previously been described. Some researchers have described complex vascular surgical maneuvers like encasement of the canine CCA in carotid siphon replicas. ${ }^{2,3}$ Also methods for generating tortuosity have been described by surgical interposition of the Dacron vascular graft (E.I. du Pont de Nemours and Company, Wilmington, Delaware) between the common trunk and the CCA, creating a vascular redundancy, which can be positioned and sutured in place to create tortuosity simulating the siphon. ${ }^{4}$ These vascular procedures are considerably challenging technically, requiring a skilled surgical team and a recovery period before device testing. Animal models with siphon anatomy resembling humans exist in the nonhuman primates; however, ethical and financial considerations preclude their use for endovascular device testing. ${ }^{5}$ 
Average Curvature

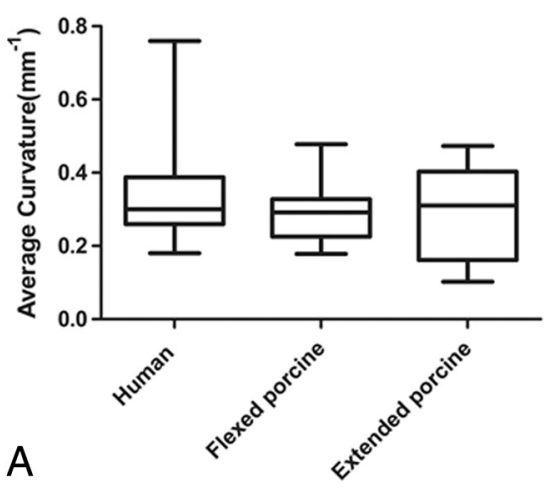

Path Length

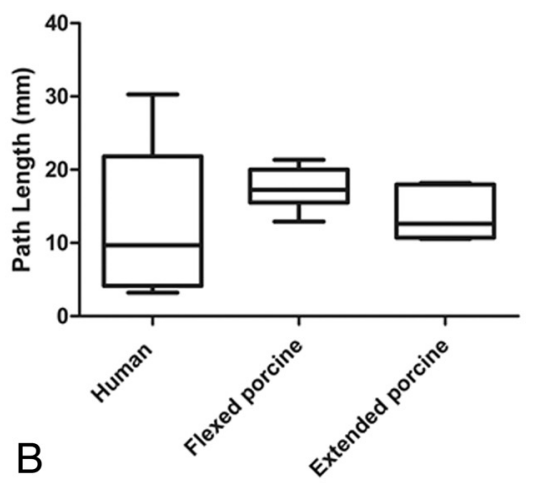

Vascular Diameters

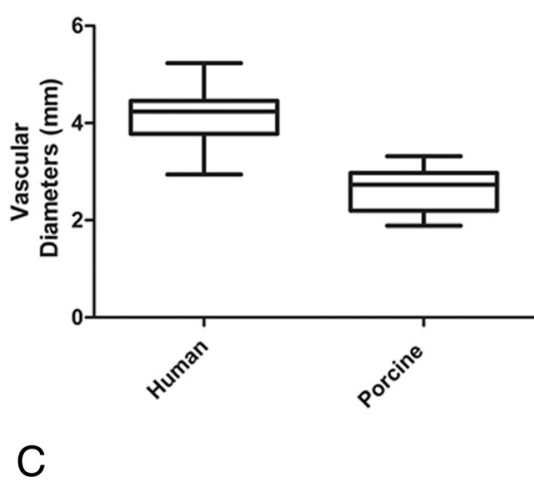

FIG 2. Box and whiskers plots showing the median, maximum, minimum, and upper and lower quartiles of the average curvature (A), path length $(B)$, and the vascular diameters $(C)$ of the human carotid siphon and the porcine brachial artery in the flexed and extended position.

Our model allows a rigorous testing of endovascular devices in a challenging tortuous vascular environment, in a model that is easily reproducible and technically much less challenging. Device performance can be evaluated on the basis of both the qualitative experience of the device while negotiating the tortuous vasculature and the angiographic response secondary to navigation. The technique for inducing the tortuosity is very feasible and reproducible and requires no prior surgical preparation. We have successfully used this vascular territory to evaluate commercially available versions of guidewires, microcatheters, endovascular stents, and their delivery systems. Further validation of the model was provided by an evaluation of the safety and performance profile of final device prototypes, before their incorporation as commercially available products for clinical use, by an experienced neuroradiologist.

Although this model is appropriate for an acute assessment, the tortuous vascular path is positional; repositioning and manipulating the forelimb may likely alter the geometry of the endovascular implants over the bends. The caliber of the target vessel does not replicate the human ICA. The porcine arteries also have a higher propensity for vasospasm. We cannot replicate the bony encasement and the level of immobility experienced at the human carotid siphon. Nevertheless, despite these limitations, endovascular device testing in this territory provides a robust environment for a rigorous assessment of the delivery and deployment of endovascular devices in a technically less demanding and reproducible in vivo model.

Disclosures: Sarena Carniato-UNRELATED: Employment: Stryker, Comments: employed full-time. Ajay Wakhloo-RELATED: Consulting Fee or Honorarium: Stryker, Comments: serve as a consultant on a per-hour basis; UNRELATED: Consultancy: Codman, Stryker, Soteira, Comments: consultation fee on per-hour basis; Grants/ Grants Pending: Philips; Stock/Stock Options: Surpass Medical. Matthew GounisUNRELATED: Consultancy: Codman, Micrus, Soteira, Comments: fee per-hour consultation; Grants/Grants Pending: Boston Scientific, Concentric, Codman, Geurbet, ev3, Micrus, Neurointerventional Therapeutics, Neuravi, Stryker, Sanofi-Aventis, Thrombolytic Scientific.

\section{REFERENCES}

1. Chueh JY, Wakhloo AK, Gounis MJ. Neurovascular modeling: small-batch manufacturing of silicone vascular replicas. AJNR Am J Neuroradiol 2009;30:1159-64

2. Tan HQ, Li MH, Zhu YQ, et al. Surgical construction of a novel simulated carotid siphon in dogs. J Neurosurg 2008;109:1173-78

3. Xie J, Li MH, Tan HQ, et al. Establishment of an experimental intracranial internal carotid artery model and the application in covered-stent navigability testing. AJNR Am J Neuroradiol 2009;30:1041-45

4. Georganos SA, Guilbert F, Salazkin I, et al. Surgical construction of an in vivo carotid siphon model to test neurovascular devices. $\mathrm{Neu}$ rosurgery 2004;54:1239-43, discussion 43

5. Tenjin H, Fushiki S, Nakahara Y, et al. Effect of Guglielmi detachable coils on experimental carotid artery aneurysms in primates. Stroke $1995 ; 26: 2075-80$ 